\title{
Visualization of membrane RNAs
}

\author{
TADEUSZ JANAS and MICHAEL YARUS \\ Department of Molecular, Cellular and Developmental Biology, University of Colorado, Boulder, Colorado 80309-0347, USA
}

\begin{abstract}
Using fluorescence microscopy, we show that previously isolated membrane-binding RNAs coat artificial phospholipid membranes relatively uniformly, except for a frequent tendency to concentrate at bends, membrane junctions, and other unusual sites. Membrane RNAs can also be visualized as single molecules or isolated complexes by atomic force microscopy (AFM) of free RNAs on mica. Finally, RNAs can be seen within membranes by AFM of RNA-liposomes immobilized on hydrophobic mica surfaces. Monomer RNAs appear globular, as expected for small RNAs. When mixed under conditions in which RNAs bind bilayers, RNA 9 and RNA 10 combine to yield about $80 \%$ of RNAs as mainly linear oligomers of $\approx 2-8$ molecules. Once inserted in membranes, the RNAs oligomerize further, yielding larger, irregular ropelike structures that prefer the edges of altered lipid patches. These properties can be interpreted in terms of RNA-RNA loop interactions, and the RNA effects on membranes can be explained in terms of an RNA preference for irregular lipid conformations. The RNA-bilayer system poses new opportunities for combining the properties of membranes and RNA in contemporary cells, and also in the ribocytes of an RNA world.
\end{abstract}

Keywords: Phospholipid bilayer; selection; ribocyte; fluorescence; atomic force microscopy

\section{INTRODUCTION}

Cells inevitably possess high concentrations of biomolecules by laboratory standards, implying that unexpected molecular interactions will be a frequent feature of the living state. Although RNA and membrane lipids in particular may seem incompatible, review of the proven interactions for small RNAs suggests that they can bind all parts of prevalent phospholipids, glycerol, phosphorylated head groups, and even the fatty acids (Khvorova et al. 1999). Thus one predicts that RNAs should be capable of direct insertion into phospholipid membrane bilayers.

Although seemingly exceptional, there are biological arguments for such insertion. Exogenous delivery of cosmochemicals and endogenous synthetic pathways probably provided amphiphilic, membrane-forming compounds to the prebiotic environment (Deamer et al. 2002). Therefore, bilayer membranes required no biosynthesis and would have been available at the origin of life. Enclosure in vesicular compartments is arguably essential for cells to benefit from their own biochemical advances, and accordingly indispensable for Darwinian evolution (Khvorova et al. 1999; Szostak et al. 2001). However, membranes made of long-chain lipids are too impermeable for cellular survival. Even if early

Reprint requests to: Michael Yarus, Department of Molecular, Cellular and Developmental Biology, University of Colorado, Boulder, CO 803090347, USA; E-mail: yarus@stripe.colorado.edu; fax: (303) 492-7744.

Article and publication are at http://www.rnajournal.org/cgi/doi/ 10.1261/rna.5129803. cells were enclosed using short-chain lipids, which make membranes more permeable (Paula et al. 1996), indiscriminate permeability would not have been sufficient to support cellular existence. Instead, a cell needs specific, directional flows of solutes to retain intermediates and import substrates. Protein elements that accomplish this are frequent in modern cells. However, a ribocyte living before the advent of templated peptide synthesis (Yarus 2002) would have required channels, pores, and porters made of RNA.

In addition, the ribose $2^{\prime}-\mathrm{OH}$ group that enhances both shape and catalysis in RNA also renders polyribonucleotides particularly susceptible to hydrolysis. An RNA-based cellular world would have benefited from a special environment having low water activity to protect interribonucleotide linkages (Pace 1991). Such an environment may be provided by the interface between organic surfaces (membranes) or inorganic surfaces (clay minerals), and bulk water solution. For both these reasons, one might predict an early connection between the RNA world and the surface world of lipid membranes.

Following such an evolutionary rationale, RNAs that can bind, change the permeability, and disrupt phospholipid bilayers have been isolated using selection-amplification (Khvorova et al. 1999; Vlassov et al. 2001). The oligomerizing heterotrimer RNA (9:9:10) ${ }_{n}$ (Fig. 1; Vlassov et al. 2001) was one particular example, found to bind stably to the exposed face of a phosphatidylcholine vesicle. Because many RNA complexes with similar activities were isolated, with no sequences in common, many RNA structures exist 
that bind phospholipid membranes. Membrane affinity is, in this sense, a simple molecular property, so it is likely that mutation would continuously produce such RNAs. Therefore, membrane-binding RNAs could easily be captured for duty in current cells. For this reason, study of membrane RNAs might illuminate an unknown aspect of modern biology, as well as bearing on early cellular evolution.

Below, we use fluorescence and atomic force microscopy (AFM) to characterize the molecular forms and disposition of the membrane-binding RNA 9:9:10 complex (see below; Vlassov et al. 2001), both free and within a phospholipid membrane.

\section{RESULTS}

\section{Binding of membrane RNAs to giant lipid vesicles}

To help visualize the membranes in our experiments, we have made large heterogeneous vesicles composed of phosphatidylcholine. We used a fluorescent lipophilic dye, Nile Red (NR), to label the membranes of these giant vesicles (GVs) during fluorescence microscopy. Nile Red is almost nonfluorescent in water solution. However, upon incorporation into the hydrophobic interior of a lipid bilayer, the dye undergoes an $\sim 40$-fold fluorescence enhancement and large absorption and emission spectra shifts to shorter wavelengths (Greenspan and Fowler 1985).

Because we wished to simultaneously determine the distribution of RNA, the green positive fluorescent cyanide dimer dye YOYO-1 was chosen to specifically visualize the membrane RNAs in the presence of lipid vesicle preparations. YOYO-1 shows a high affinity for DNA and RNA (Bleher and Martin 2001) and undergoes a large fluorescent enhancement upon intercalation into nucleotide structures.

Microscopy with double fluorescence detection effectively showed the binding of RNA complexes on the surface of giant lipid vesicles. The first column of Figures 2A,B,C
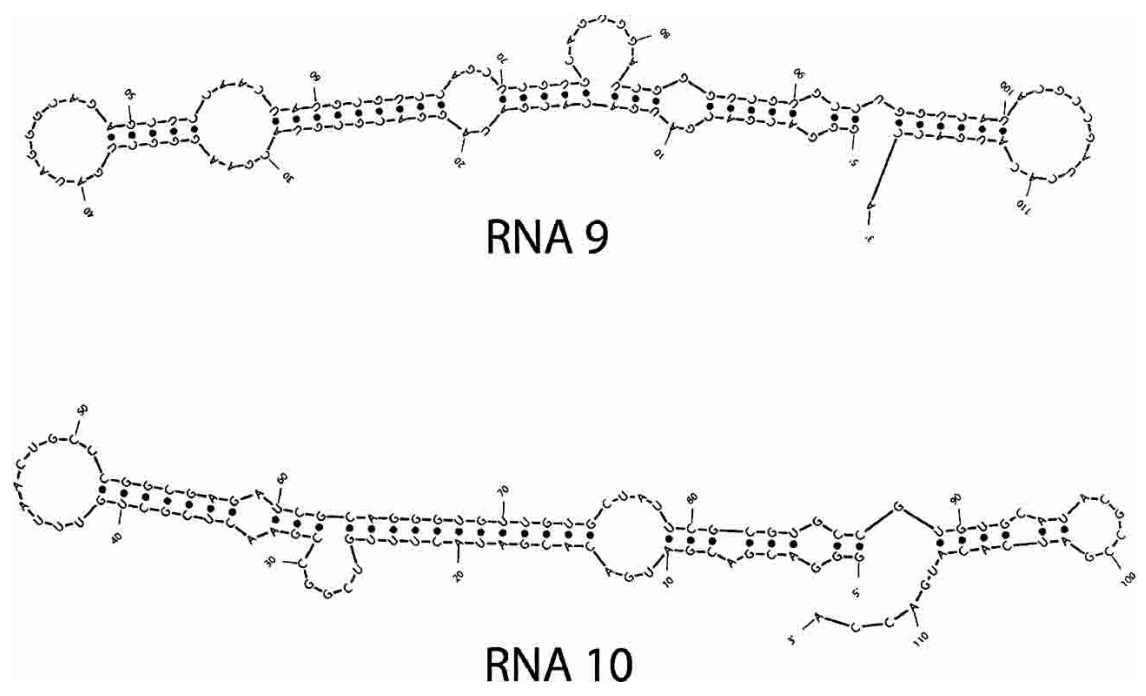

FIGURE 1. Proposed secondary structures (Vlassov et al. 2001) for RNA 9 and RNA 10. and $3 A, B$ shows a fluorescence image of vesicle membranes stained with NR (red fluorescence), and the second column of Figure 2A,B,C shows the corresponding image of RNA molecules stained with YOYO-1 (green fluorescence). The similarity of these two distributions is evident in the separate images, but the merged images in the third column of Figure 2 (the superposition of red and green gives a yelloworange color) allow close visual comparison of the lipid and RNA distributions.

As a first approximation, RNA and phospholipid intensities track very well in the third set of images. This is confirmed and quantitated in the last column (right), where red and green fluorescent intensities along the marked diagonal bars in previous images are plotted. We expect from previous biochemical measurements (Vlassov et al. 2001) that RNA 9:9:10 is stably bound to the bilayer. In these plots, we see the green signal from RNA 9:9:10 tracking the complex distribution of membrane surface across vesicles viewed at varying angles, confirming that the RNA is concentrated on and bound to the phospholipid membrane.

However, observed at finer resolution, there are small differences between lipid and RNA. The simplest difference is that in the original images and plots, a low level of green light can be seen as a background, away from the vesicles (Fig. 2A,B,C, sides). These green quanta indicate free RNA in the solution. This corresponds to the previous finding that fewer than $100 \%$ of RNA molecules bind to liposomes (Vlassov et al. 2001).

A subtler and potentially more interesting difference is that within the membrane systems, there are small areas where RNA and phospholipid do not vary proportionately. Green "caps" in the third column of Figure 2 suggest accumulation of RNA molecules in specific regions of vesicles. This can be seen at the neck of the inner vesicle on the top right in Figure 2A, at the neck of the vesicle junction in Figure $2 \mathrm{~B}$, and at the poles of the large vesicular compartment in Figure 2C. One way to summarize this tendency is that the RNA complexes tend to be concentrated on highly curved parts of the membrane or at places where there are complex membrane intersections. We will return below to this tendency for the RNA 9:9:10 complex to prefer particular membrane regions, which may be interpretable in molecular terms.

Figure $3 \mathrm{~A}, \mathrm{~B}$ shows control experiments for GVs/RNA 9 alone and GVs/ RNA 10 alone, respectively. Red and green cross sections along the bars are presented in the fourth column of Figure 3. Lower relative green intensities for RNA indicate a large drop in the binding of single RNA molecules, compared to the RNA 9:9:10 consortium. In 

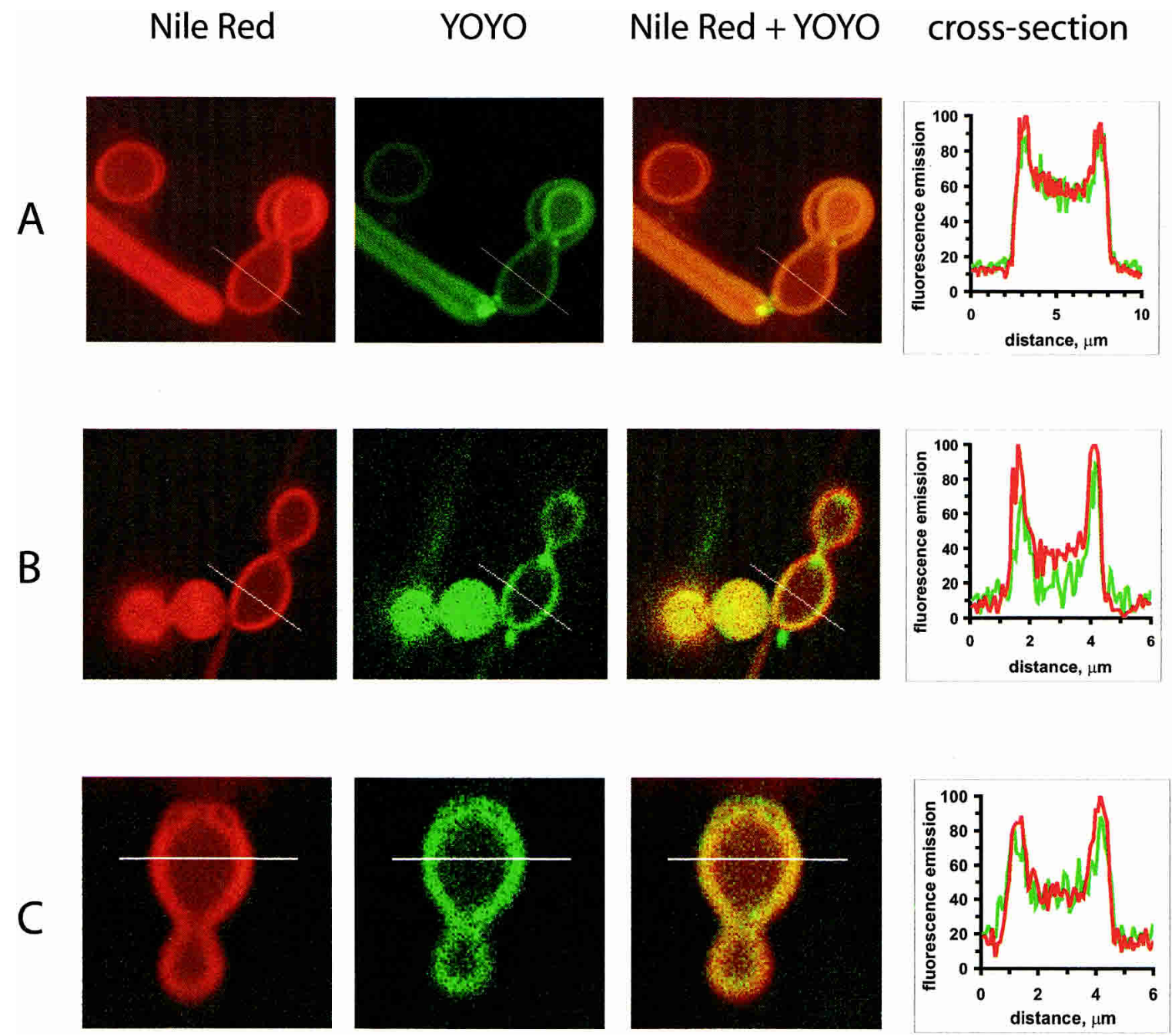

FIGURE 2. Fluorescence microscopy $(A, B, C)$ of large lipid vesicles (membrane visualized by Nile Red) incubated with RNA 9:9:10 complexes at RNA 9/RNA 10 mole ratio equal 2 (RNA visualized by YOYO-1), and fluorescence intensities along the diagonal bars.

another series of control experiments (not shown), we have analyzed images of GVs/NR/YOYO without added RNA. These experiments showed a weak green image of the vesicular system, very much like that in the second column of Figure 3A,B, despite the complete absence of RNA in these experiments. The same kind of weak image in the green channel can be seen with NR and vesicles alone, despite the fact that neither RNA nor YOYO is present. Accordingly, there is an $\sim 3 \%$ crossover from the NR red to the YOYO green channel due the imperfect separation of the spectra by our microscope's optical system. In the other direction, controls with YOYO as the only fluor showed no detectable red signal. Therefore, for quantitative purposes, micrographs like Figures 2 and 3 should be corrected by subtraction of the $3 \%$ green crossover intensity due to the red lipid fluor to get a corrected RNA (green) distribution.

When we subtracted crossover intensities from Figure 3A,B (GVs/RNA 9 alone and GVs/RNA 10 alone, respectively), there was essentially no significant binding of RNA
10 to vesicles when no RNA 9 was present. This is consistent with biochemical indices, which gave no evidence of RNA 10-membrane affinity (Vlassov et al. 2001). With crossover into the green channel subtracted, there was marginal but perhaps significant $(\leq 1 \%)$ binding of RNA 9 alone on the surface of vesicles, when compared to its binding in the presence of RNA 10. This is consistent with one's (unnormalized) impression of the green images themselves (Fig. 3). If accepted as a significant signal, these findings would be consistent with previous biochemical data, indicating a weak or transient interaction between liposomes and RNA 9 alone (Vlassov et al. 2001). But whether crossover corrections are made or not, these fluorescent images confirm a requirement for both RNA 9 and 10 to observe full membrane affinity.

\section{Binding of membrane RNAs to mica surfaces}

Mica belongs to a group of clay minerals. These minerals are layered silicates, the layers being stacked on top of each 


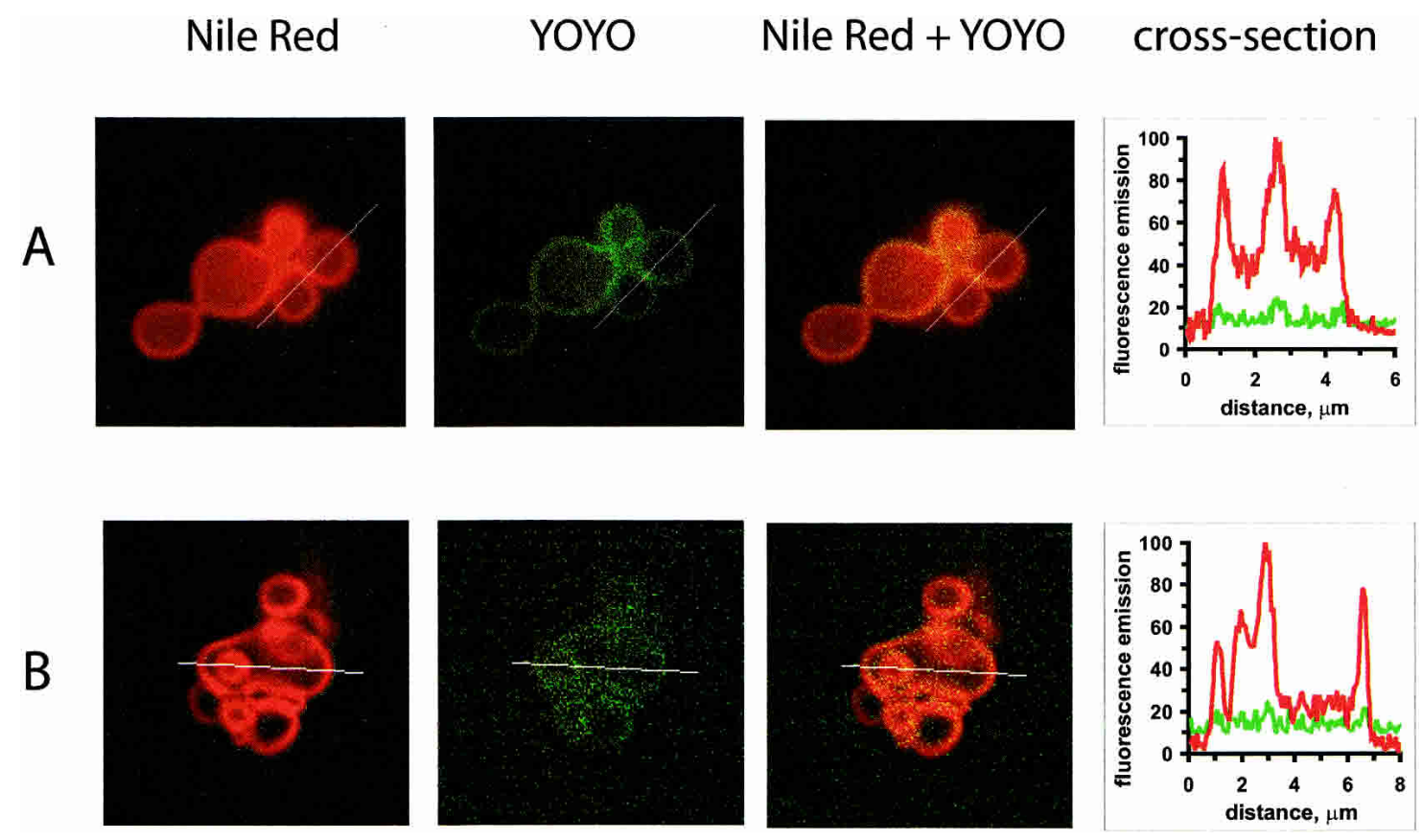

FIGURE 3. Fluorescence microscopy of large lipid vesicles (visualized by Nile Red) incubated with RNA 9 ( $A$ ) and RNA 10 (B; visualized by YOYO-1), and fluorescence intensities along the diagonal bars.

other (Środoń 1999). By cleaving between layers, one easily obtains thin atomically flat sheets with a negative surface charge (Hansma and Laney 1996). Nucleic acids can be immobilized on mica surfaces in the presence of $\mathrm{Ni}^{2+}$ cations (Hansma and Laney 1996). With $\mathrm{Mg}^{2+}$ cations only, nucleic acids do not bind tightly enough to mica for AFM imaging. However, $\mathrm{Ni}^{2+}$ cations have an anomalously high enthalpy of hydration that may allow them to form strong complexes with ligands other than water (Hansma and Laney 1996). Thus $\mathrm{Ni}^{2+}$ cations probably function as salt bridges mooring negatively charged DNA/RNA chains to the hydrophilic, negatively charged, aluminosilicate surface of muscovite mica. The millimolar levels of $\mathrm{Ni}^{2+}$ required (see Materials and Methods; $\mathrm{Mg}^{2+}$ and $\mathrm{Ca}^{2+}$ are also present) to see the RNAs by AFM are a potential perturbation for an RNA tertiary structure. However, $\mathrm{Ni}^{2+}$ as used in AFM imaging does not interfere with binding of RNA 9:9: 10 to liposomes, determined by exclusion chromatography (T. Janas and M. Yarus, unpubl.). Furthermore, the intermolecular RNA interactions we expect occur readily (see below). Thus the $\mathrm{Ni}^{2+}$ required for AFM imaging does not observably perturb these RNAs.

We have visualized membrane RNAs on such mica surfaces using tapping mode AFM. This technique, in which the position of a sharp oscillating probe is followed by reflected laser light while the probe scans a surface for particles, is very accurate for measurements of the height of a microscopic object. However, in the horizontal plane, the objects have increased apparent dimensions due to "tip broadening." That is, as the probe tip scans over the object, the tip radius is added to both sides (Sedin and Rowlen 2001). Thus object height is reliably measured, but molecular details are somewhat submerged in a broadened molecular image.

A control image for mica treated with buffer plus $\mathrm{Ni}^{2+}$ but without RNA, is presented in Figure 4A. Quantitative analysis of such images shows mean vertical excursions of a few tenths of a nanometer, imaged as color differences in the figure. These fluctuations can arise from adsorption of hydrated divalents on the mica surface. The radius of the fully hydrated $\mathrm{Ni}^{2+}$ ion is $\sim 0.2 \mathrm{~nm}$ (including inner shell water; Marcus 1985). In addition, the mica surface has cavities because its hydroxyl groups are recessed slightly (Hansma and Laney 1996). This small and reproducible surface roughness does not disturb our measurements because 113-118-nt RNAs are objects 5-10-fold taller and greater in breadth (below).

Figure 4B shows AFM images of RNA 9 alone, Figure 4C shows RNA 10 alone, and Figure 4F shows the result of mixture of a ratio of 2 moles of RNA 9 to 1 mole RNA 10 . The nature of the particle height distributions in these experiments can be judged from Figure $4 \mathrm{D}$, which shows the measured AFM particle heights for the molecules of RNA 10 pictured in 4C. All RNA structures showed a similar height distribution to that shown here, with moderate asymmetry, finite variance, but a single mode with a well-defined modal value for height. Thus below we have used modal heights as an index for the various RNAs.

Adsorbed RNA 10 appears as a mostly homogeneous distribution of independent particles. Fully extended, RNA 10 

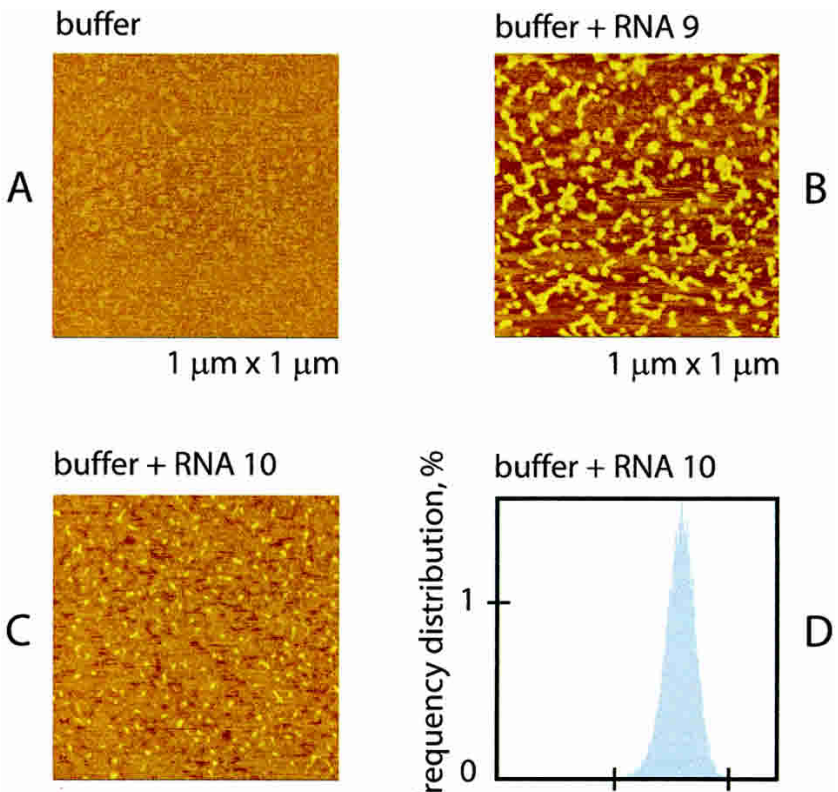

$1 \mu \mathrm{m} \times 1 \mu \mathrm{m}$

buffer +

RNA 9 with AU loop mutation

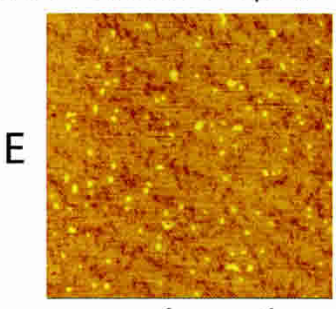

$1 \mu \mathrm{m} \times 1 \mu \mathrm{m}$

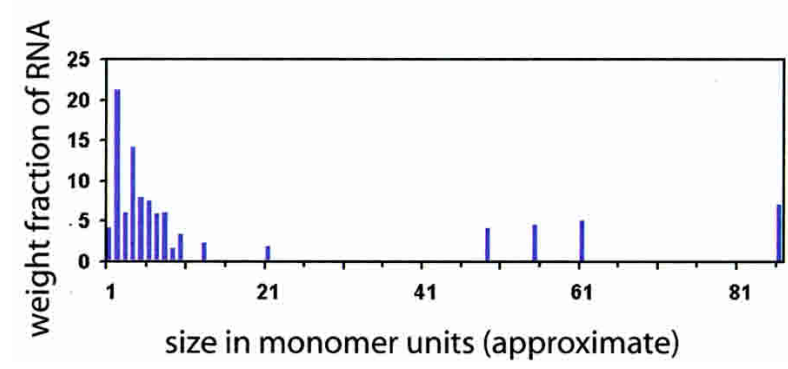

FIGURE 4. Atomic force microscopy images of mica surface incubated with buffer $(A)$, RNA $9(B)$, and RNA $10(C)$; frequency distribution of the height of RNA $10(D)$; AFM images of mica surface incubated with RNA 9 with AU loop mutation $(E)$ and RNA $(9+10)$ at RNA 9/RNA 10 mole ratio equals $2(F)$; and distribution of the weight fraction of RNA aggregates in panel $\mathrm{F}(G)$. Below each AFM panel, the exact size of the field is indicated so particles can be sized.

would be a thin rod more than $9 \mathrm{~nm}$ long (Fig. 1). Tip broadening (see Materials and Methods) would extend this into an ovoid about $20 \times 30 \mathrm{~nm}$, somewhat dependent on the profile of individual tips and on the molecular orientation with respect to the AFM scan. Just such ovoids are the major form observed in images of free RNA 9 and RNA 10 molecules (e.g., Fig. 4C,E). Thus, free RNA 10 is about the size expected of a single RNA molecule (Fig. 4C), with a modal height of $1.7 \mathrm{~nm}$ (for scale, an uncompressed RNA A-form helix is $2.1 \mathrm{~nm}$ in diameter). These AFM results accordingly agree well with the predominant monomers of free RNA 10 observed by native gel electrophoresis (Vlassov et al. 2001).

In contrast (Fig. 4B) RNA 9 appears as a set of irregular aggregates ranging from apparent monomer size to long chains. These are taller than RNA 10 (although the secondary structures of the monomers are similar; Fig. 1) at about $2.5 \mathrm{~nm}$. These chained, mostly linear RNA 9 aggregates require the structure of the "right-hand" loop (compare Fig. 1 ), because mutation of $2 \mathrm{nt}$ in the right-hand loop (GA $\rightarrow$ AU; Vlassov et al. 2001) disrupts aggregation (detected on gels) and liposome affinity (detected by chromatography). The disruption of RNA oligomerization for the mutant RNA can also be seen by AFM in Figure 4E, where the GA $\rightarrow$ AU mutant RNA 9 reverts completely to free monomers on the mica surface. The modal height of these monomers is $\sim 1.4 \mathrm{~nm}$. Thus the greater height of the chained aggregates reflects the changed (more rigid, taller) structure of oligomerized RNA 9, because the mutation that disrupts oligomerization results in measured heights similar to the free RNA 10 monomer (above; the relatively small AU loop mutation should have no large-scale structural effect). This ability of RNA 9 to aggregate extensively dependent on its right-hand loop, evident in Figure 4C, will be important below when we discuss larger aggregates.

Finally, in Figure 4F, we have the mixture of RNA 9 and RNA 10. These appear as varied short oligomers (compare to Figure 4B,D above), with an occasional much larger aggregate. The predominant short oligomers are mostly linear, but large aggregates can have complex closed forms containing apparent loops and nets. To quantitate these observations, the field in Figure 4F was scored for the number of monomers (taken to be an ovoid about $30 \mathrm{~nm}$ in major dimension) within each particle resolved within the field. Because individual molecules are not resolved within RNA oligomers by AFM, there is a clear element of subjectivity in this scoring, and so only the gross aspects of the resulting distribution are interpretable. However, even a rough size distribution yields essential conclusions.

Size distribution results are plotted in Figure 4G, as the weight fraction on the ordinate (number of particles $\times$ size in monomers) versus approximate size in monomer units. An easily detectable fraction of RNA appears unaggregated; $4 \%$ appear to be monomers. Most significantly, RNA oligomers usually appear small and linear. For example, oligomers apparently containing from 2 through 8 monomer RNAs represent $67 \%$ of total visible RNA particles and $80 \%$ of all RNA monomers. However, there is a long tail toward large aggregates-these, though numerically few, represent a substantial weight fraction of total RNA (particles with $>8$ monomers are $29 \%$ of RNA in the figure). This distribution seems consistent with native gel electrophoresis (Vlassov et al. 2001), where the RNA was concentrated at small sizes 
but showed a long tail smeared to higher aggregates within which separate molecular forms were not resolved. These aggregates have a modal height of $\sim 2.4 \mathrm{~nm}$, greater than either monomer, again suggesting a taller or more rigid joint structure. The strong concentration on short oligomers among these free RNAs will be important below because membrane-bound RNA structures appear quite different.

\section{Binding of membrane RNAs to a hybrid lipid bilayer}

We have used lipid bilayers supported on mica to visualize RNA in its apparent membrane-bound form, again using tapping mode AFM. Hybrid lipid bilayers were formed in two consecutive steps: First, a hydrophobic surface was created using mica coated with a thin layer of gold. The gold is used to support a hydrocarbon layer formed using the thiol group of octadecanethiol. The hydrocarbon, moored through the affinity of its thiol for the heavy metal gold, orients toward the air phase. Then liposomes were applied to this surface in suspension; these spontaneously fused with the hydrophobic octadecanethiol layer and, perhaps, with each other. The hydrocarbon chains of octadecanethiol and phosphatidylcholine molecules form a hybrid bilayer next to the mica-gold substrate. Figure 5A,B shows chains of 100-200-nm vesicles fused to the gold-hydrocarbon surface in the presence of RNA 9 alone and RNA 10 alone, respectively. The phospholipid surface appears completely clean, indicating that no RNA molecules are bound when only RNA 9 or RNA 10 was present. These vesicles, in fact, appear identical to control vesicles fused onto the same
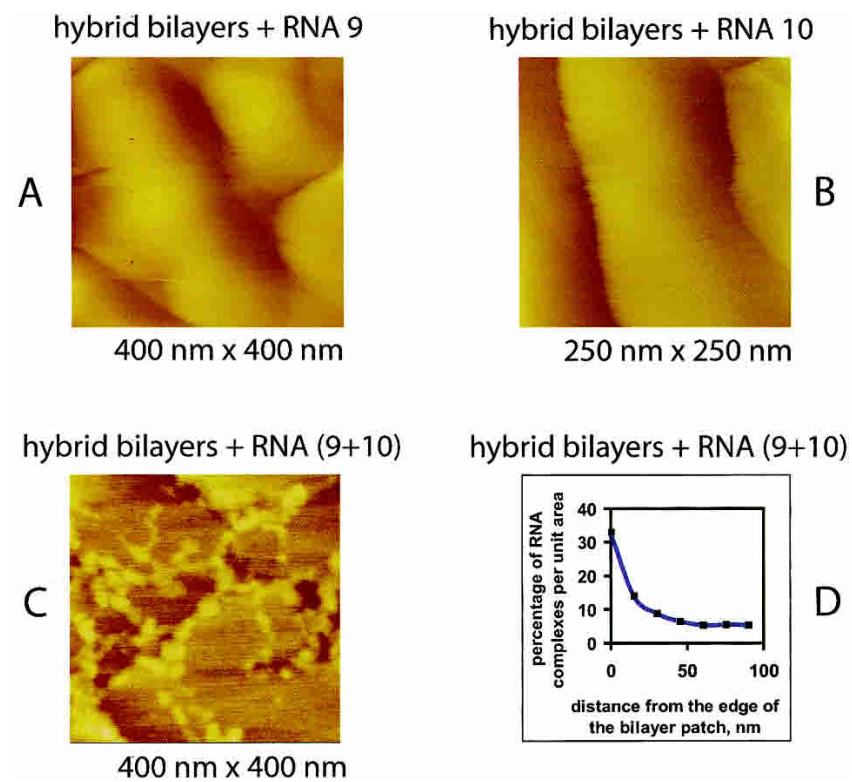

FIGURE 5. Atomic force microscopy images of hybrid lipid bilayer surface incubated with RNA $9(A)$, RNA $10(B)$, and RNA $(9+10)$ at RNA 9/RNA 10 mole ratio equal $2(C)$, and percentage of area as RNA $(9+10)$ complexes as a function of the distance from the edge of a patch of bilayer $(D)$. Below AFM panels, the full size of the field is indicated so objects may be sized. octadecanethiol-gold-mica surface without exposure to RNA at all (not shown).

As judged from the AFM height measurements of fused bilayers, vesicles appear to be partially fused to the goldhydrocarbon surface. There are regions (lightest color) having a thickness of two bilayers (about $10 \mathrm{~nm}$ ) and regions (darker color) having a thickness of one bilayer (about 5 $\mathrm{nm}$ ). The darkest regions, outside the vesicles, are presumably covered only by octadecanethiol molecules. Within the regions of $10 \mathrm{~nm}$ height, the vesicles have probably fused through their lower bilayer, and a hybrid octadecane-phospholipid membrane is underneath (perhaps as a patchwise fusion) with a pure phospholipid membrane on top.

When complexes of RNA 9:9:10 are applied to the vesicles adsorbed on coated mica (Fig. 5C) before AFM, irregular ropes of RNA are reproducibly seen at the periphery of flattened phospholipid membranes. Lipid regions themselves are flattened and have more irregular edges, seemingly defined by the RNA aggregates. These changes in lipid distribution are apparently an effect of RNA binding.

There are two unexpected aspects of the appearance of membrane-bound RNA 9:9:10. First, membrane-bound RNA chains are much greater in diameter and longer in length than the same RNAs free on a mica surface. Even allowing for possible changes in RNA conformation when bound, it seems safe to say that RNA aggregates to greater extents within the membrane, compared to the oligomers on mica (cf. Fig. 4F). Second, RNA molecules appear to be associated with the edges of the bilayer patches, and are almost absent from central regions of lipid. Figure 5D shows the relative density of RNA molecules versus their distance from the edge of the bilayer. The apparent surface density of RNA molecules located at the edge is $\sim 6 \times$ the density far from the edge $(60-90 \mathrm{~nm})$.

\section{DISCUSSION}

We have visualized previously isolated membrane RNAs by light and AFM microscopy. The RNAs behave in a manner clearly related to that inferred from molecular biology/biochemistry data. Most notably, using fluors that label both lipid and RNA, the RNA and lipid distributions track through space across vesicles of a variety of complicated shapes. The only evident way for RNA to reproducibly do this is to be inserted in or bound to the phospholipid membrane. Thus the liposome-binding selection yielded supramolecular RNA complexes, which bind stably to the surfaces of phosphatidylcholine liposomes. This had been previously suggested by co-chromatography of RNA and liposomes and RNA effects on membrane permeability (Vlassov et al. 2001). For example, we previously showed that these RNA consortia caused release of liposome-encapsulated solutes and also disrupted planar lipid membranes. Thus, a variety of experiments agree that these RNAs stably bind to phospholipid membranes and alter their properties. 
In addition, the codistribution of YOYO fluorescence with lipid depends on the presence of both RNA 9 and RNA 10, as was suggested from the biochemical studies. Therefore strong membrane affinity by these selected RNAs is a property of consortia rather than single RNAs (Vlassov et al. 2001).

\section{Structure of a membrane RNA}

AFM imaging of RNAs on mica suggests that RNA 10 monomers and RNA 9 aggregates rearrange when mixed. Free RNA 9:9:10 appears in AFM as approximately twothirds short, linear oligomers containing 2-8 monomer RNAs. Remaining oligomerized RNA molecules appear in much larger heterogeneous aggregates with varied shapes, comprising $29 \%$ of RNA. Similar oligomerization was also evident on native gels (Vlassov et al. 2001), occasionally ranging upward to RNA aggregates that would not enter agarose gels. Taking from the present AFM evidence the idea that the predominant form of RNA 9:9:10 is short oligomers, both numerically and by weight, we can greatly constrain such small oligomeric structures using previous evidence.

First, lead and S1 nuclease probing of RNA 9 and RNA 10 (Vlassov et al. 2001) was consistent with calculated stable secondary structures, which feature extended bulged helices bounded by loops (Fig. 1). Analysis of the loops revealed complementary antiparallel sequences at the "left" in RNA 9 (GGGCAG, nt 42-48) and RNA 10 (CUGCCC, nt 47-52; Fig. 1). These leftward hexanucleotide loop sequences apparently hybridize, giving a chemical protection signal when RNAs are mixed. This is characteristic of a "kissing loop complex" (Tomizawa 1984). In addition, the rightward loops of RNA 9 and RNA 10 contain a self-complementary tetranucleotide GAUC (nt 106-19 and 101-104 for RNA 9 and RNA 10, respectively). Probing, oligonucleotide, and mutation experiments suggest that RNA 9 and RNA 10 can also interact using these complementary rightward loop GAUCs (Figs. 1, 4E).

Therefore only 9r:9r, 10r:10r, and 91:10l (where $\mathrm{r}$ and 1 indicate the right and left loops, respectively, in Fig. 1) are sequence-allowed loop:loop interactions for which there is independent evidence. However, though the 9r:9r intermolecular interaction probably exists (Fig. 4B; Vlassov et al. 2001), pure RNA 10 is a stable monomer (Fig. 4C; Vlassov et al. 2001). Thus, only 9r:9r and 101:91 interactions (and possibly interactions between the rightward loops of RNA 9

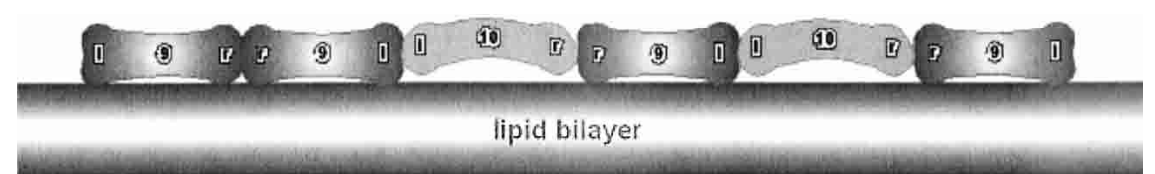

FIGURE 6. Hypothetical small RNA 9:9:10 oligomer consistent with prior biochemical data on sequences and stoichiometry, and also consistent with present microscopy. The simplest hypothesis posits this structure in free RNA as well as within membrane-bound RNAs. and 10) are stable. Given that consortium stoichiometry is also close to 2 moles of RNA 9 for each RNA 10 (Vlassov et al. 2001), only a few types of small aggregate close to the sizes visualized by AFM can be rationalized. These allowed forms are shown as a hexameric aggregate in Figure 6. In the figure, one allowed type of trimer is shown on the left and the other is shown on the right, prospectively joined through the right-hand tetrameric self-complementarity at the middle. A mixture of these interactions, or these with small modifications (e.g., RNA 10 could be added to the right and left ends shown) could explain the AFM appearance of RNA 9:9:10 (Fig. 4F). However, the distribution of sizes is puzzling in one respect; we cannot presently account for the fact that these smaller aggregates are occasionally dramatically extended to give the large, complex aggregates observed when mixed RNA 9 and RNA 10 are displayed on mica. This may, however, be an essential reaction-although large forms are rare when considered as a number fraction, because of their size they represent a significant fraction $(\approx 29 \%$ ) of total RNA (Fig. $4 \mathrm{G})$. Thus, it is numerically possible that the occasional large forms are the only forms bound to the membrane surface or that they uniquely initiate the membrane-bound RNA structures.

AFM suggests striking changes when RNA 9:9:10 enters a phospholipid bilayer. Shorter aggregates (Fig. 4F) become larger, highly irregular chained structures (Fig. 5C), which seem to have dramatically aggregated laterally and lengthwise. Such further RNA oligomerization may perhaps use the capacity for extensive complex formation through right hand loops of RNA 9, as seen in our AFM images (e.g., cf. Fig. 4C). However, it is possible that this second, membrane-specific RNA oligomerization will use new interactions unique to the RNA-phospholipid domain.

\section{Effects of membrane RNA on lipids}

As is evident in Figure 5C,D, the large irregular RNA aggregates in the membranes appear at the boundaries of flattened lipid patches. This observation recalls the fluorescent micrographs where the RNA tended to concentrate at points where the bilayer is bent or otherwise disturbed (Fig. 2 ). This molecular preference can arise very simply, given that the RNA probably binds phospholipid (via RNA 9). These membrane RNAs prefer areas where the structure of the lipid leaflet is irregular, where a bulky RNA can get less hindered access to its ligand, dioleoylphosphatidylcholine.

By the same token, the RNA will stabilize pores by stabilizing the unusual lipid conformations at their edges. Permeation across a bilayer membrane can occur through hydrated transient defects produced by thermal fluctuations within the bilayer (Paula et al. 1996). By passing through such hydrated pores, a permeating ionic molecule can avoid the 
Born high-energy barrier associated with partitioning into the hydrophobic membrane interior. The membranebound RNA may stabilize just such disturbances in the membrane lipid to produce increases in permeability.

This notion also explains data showing that RNA 9:9:10 disrupts a black lipid membrane and releases GTP encapsulated in liposomes (Vlassov et al. 2001). Thus a combination of RNA and lipids can create a microdomain within the membrane, which has unique conformational preferences. Some fluorescence micrographs suggest other kinds of possibly related intermediates, as in Figure 2A,B,C, where an unusual concentration of RNA appears at the narrowed neck of a vesicle, apparently pinching into two compartments. This somewhat parallels the conformational effects of other lipids on phospholipid bilayers, as for polyisoprenol-DOPC bilayers (Janas et al. 2000).

This model for membrane RNAs explains the need for selection to observe membrane affinity, despite the fact that the sequences employed must be very simple. On the one hand, at least one RNA of a membrane consortium must have membrane affinity. Based on our experiments, this is likely RNA 9. However, RNA 10, which has never shown detectable lipid affinity, is also required for strong binding. We posit that RNA 10 is a linker selected to poise adjacent RNA 9 molecules so that their phospholipid affinity is directed mostly to one side. In other words, the complex can reach the flat lipid surface from multiple places, especially within the huge aggregates that exist on vesicle surfaces (compare Fig. 6). Sided phospholipid affinity would be rare among individual randomized RNA molecules, and require substantial selection, as observed (Khvorova et al. 1999; Vlassov et al. 2001).

We have observed an unanticipated variety of phenomena given that liposome affinity was the only RNA property initially selected during isolation of these RNAs. Selected RNAs provoke greater permeability to ions and larger polar molecules (Vlassov et al. 2001) as well as a crude sort of membrane morphogenesis (this work). These observations point to the speculative possibility that membrane RNAs might have an unexpected range of effects on the shape, permeability, and structural preferences of biomembrane systems, and could thereby participate more broadly in cellular life than previously envisioned.

\section{Membrane RNAs and cells}

Given that many selected RNA sequences are observed to bind a membrane composed of a prevalent biological lipid, and that the requirements for functional consortia seem to be easily reproduced, the question of whether membrane RNAs exist in modern cells arises. On one hand, there are modern RNAs that show unexplained association with intracellular membranes, for example, in the case of Escherichia coli signal recognition particle (Batey et al. 2000; Walter et al. 2000). In addition, mutant phenotypes suggest the involvement of membrane RNAs in the permeability of the Saccharomyces cerevisiae cell membrane (MacIntosh et al. 2001). However, Figure 5C suggests the participation of many RNA molecules in the lipid-bound form of RNA. An unavoidable requirement for such large structures would probably restrict biological use of this RNA property. However, the AFM images do not allow strong conclusions about what is required for membrane affinity, because there are frequent, apparently small aggregates (Fig. $5 \mathrm{C}$ ) visible in the membrane. The smallest stably bound membrane RNA consortia therefore become an interesting subject for further investigation.

\section{MATERIALS AND METHODS}

\section{Preparation of membrane RNAs}

RNA sequences previously selected for binding to phosphatidylcholine liposomes (Vlassov et al. 2001) and designated RNA 9 and RNA 10 (Fig. 1) were heated in water for $3 \mathrm{~min}$ at $65^{\circ} \mathrm{C}$, then $10 \times$ buffer was added (buffer: $50 \mathrm{mM}$ HEPES at pH 7.0, $50 \mathrm{mM} \mathrm{NaCl}$, $5 \mathrm{mM} \mathrm{MgCl}_{2}, 2 \mathrm{mM} \mathrm{CaCl}_{2}$ ), and the solution was cooled to room temperature over $10 \mathrm{~min}$. The RNAs and their mixtures were analyzed using $8 \%$ polyacrylamide gel electrophoresis and $2 \%$ agarose gel electrophoresis performed in $50 \mathrm{mM}$ HEPES ( $\mathrm{pH} 7.0$ ), 50 $\mathrm{mM} \mathrm{NaCl}, 5 \mathrm{mM} \mathrm{MgCl} 2$, and $2 \mathrm{mM} \mathrm{CaCl}_{2}$.

\section{Preparation of giant lipid vesicles}

Giant vesicles used for fluorescence microscopy were prepared using a procedure described by Yang and Glaser (1996). The phospholipid (DOPC, 250 nmoles) in $\mathrm{CHCl}_{3}$ and the fluorescent phenoxine dye Nile Red [ 0.5 nmoles (Sigma), emission $\lambda_{\text {max }} \approx 640$ $\mathrm{nm}$ when incorporated in phospholipid bilayer] in ethanol were mixed and placed in a small glass vial $(15 \mathrm{~mm} \times 45 \mathrm{~mm})$, and dried using a weak stream of nitrogen to a thin film along the bottom of the vial, then the vial was placed for $2 \mathrm{~h}$ in vacuum to remove residual solvents. The DOPC/NR thin layer was hydrated slowly in $500 \mu \mathrm{L}$ of the buffer. Giant vesicles $(2-10 \mu \mathrm{m})$ were obtained from the upper layer of the solution, after a minimum 48 -h incubation at room temperature in dark.

\section{Fluorescence microscopy}

Membrane RNAs at a final concentration of $87 \mathrm{nM}$ (RNA 9 alone, RNA 10 alone, RNA 9/RNA 10 mole ratio equals 2) in the buffer were mixed with the cationic cyanide fluorescence dye YOYO-1 iodide [final concentration $910 \mathrm{nM}$ (Molecular Probes), emission $\lambda_{\text {max }} \approx 510 \mathrm{~nm}$ when bound to RNA). At these concentrations, there were 11 RNA nucleotides/dye molecule.

The suspension of DOPC/NR giant vesicles and the solution of RNA/YOYO were gently mixed at a volume ratio of 10:1, and then mounted on glass coverslips. The images were collected after $\sim 2 \mathrm{~h}$ on a Zeiss Axioplan2 fluorescence microscope (Carl Zeiss, Jena) with a $100 \times$ Plan-APO objective lens (numerical aperture 1.4). Images were recorded with a Cooke SensiCam charge-coupled device camera and processed with the use of the SlideBook software package (Intelligent Imaging Innovations). 


\section{Atomic force microscopy of membrane RNAs on mica}

Membrane RNAs were folded in buffer at total concentrations of $30 \mu \mathrm{M}$ and then diluted in buffer to a final concentration of 14 $\mathrm{nM}$. Ten $\mu \mathrm{L}$ of RNA solution containing $3 \mathrm{mM} \mathrm{NiCl}_{2}$ were deposited onto freshly cleaved mica, and after 2 min the mica surface was rinsed with water and dried with a weak flux of nitrogen (Rivetti et al. 1999). The 3-mM concentration of $\mathrm{Ni}^{2+}$ was found optimal for binding of RNA molecules to the mica surface (Fay et al. 2001). AFM images were obtained in air with a Nanoscope III microscope (Digital Instrument, Inc.) operating in the tapping mode. High Aspect Ratio Etched Silicon Probes (Digital Instrument) of resonance frequency $\sim 276 \mathrm{kHz}$ were used. The supplier says of these probes: "The tip radii are typically better than $10 \mathrm{~nm}$. We guarantee at least $15 \mathrm{~nm}$." Water was obtained from an Ultrapure Water System Milli-Q Plus (Millipore).

\section{Preparation of hybrid bilayers}

Alkanethiol monolayers were prepared by immersing gold-coated mica substrates (Molecular Imaging) in 1-mM octadecanethiol (Aldrich; 98\%) solution in pure ethanol (Aaper Alcohol and Chemical Co.) at room temperature for a minimum of $12 \mathrm{~h}$ (Bain et al. 1989), and then the surface was washed with ethanol. Increase of the contact angle between this surface and the surface of a drop of water was dramatic, indicating the formation of a hydrophobic surface (Bain et al. 1989).

Unilamellar liposomes were prepared from phosphatidylcholine (DOPC; 1,2-dioleoyl-sn-glycero-3-phosphocholine; Avanti Polar Lipids) at a final concentration of $20 \mathrm{mg} / \mathrm{mL}$, by using the Avanti MiniExtruder with pore filter diameter of $400 \mathrm{~nm}$ (Vlassov et al. 2001).

Hybrid bilayer membranes (HBMs) were formed by applying the suspension of DOPC liposomes on the alkanethiol monolayer for $90 \mathrm{~min}$ to allow the reorganization of the lipid vesicles and the formation of the bilayer (Meuse et al. 1998), and then the surface was washed with water. Decrease of the contact angle between this surface and the surface of a drop of water was clear, indicating the formation of a hydrophilic surface (Bain et al. 1989).

\section{Atomic force microscopy of membrane RNAs on hybrid bilayers}

Ten $\mu \mathrm{L}$ of RNA solution containing $3 \mathrm{mM} \mathrm{NiCl}_{2}$ were deposited onto HBM, and after $20 \mathrm{~min}$, the mica surface was rinsed with water and dried with a weak flux of nitrogen.

AFM images were obtained in air with a Nanoscope III microscope (Digital Instrument, Inc.) operating in the tapping mode. High Aspect Ratio Etched Silicon Probes (Digital Instrument) of resonance frequency $\sim 276 \mathrm{kHz}$ were used. The relative density of RNAs was calculated as the ratio of the fractional area in RNA complexes within a 15-nm-wide zone along the edge to the total fractional area of RNA complexes on the interior bilayer patch.

\section{ACKNOWLEDGMENTS}

We thank the members of our laboratory and also Dr. Kathy Rowlen and Dr. Jessica Ekhoff (University of Colorado, Chemistry), who hosted T.J.'s AFM experiments. Thanks also to Dr. J. Richard McIntosh (University of Colorado, MCD Biology) for the loan of the fluorescence microscope. This work was supported by the National Institutes of Health Grant GM 30881.
The publication costs of this article were defrayed in part by payment of page charges. This article must therefore be hereby marked "advertisement" in accordance with 18 USC section 1734 solely to indicate this fact.

Received July 16, 2003; accepted August 13, 2003.

\section{REFERENCES}

Bain, C.D., Troughton, E.B., Tao, Y.-T., Evall, J., Whitesides, G.M., and Nuzzo, R.G. 1989. Formation of monolayer films by the spontaneous assembly of organic thiols from solution onto gold. J. Am. Chem. Soc. 111: 321-335.

Batey, R.T., Rambo, R.P., Lucast, L., Rha, B., and Doudna, J.A. 2000. Crystal structure of the ribonucleoprotein core of the signal recognition particle. Science 287: 1232-1239.

Bleher, R. and Martin, R. 2001. Ribosomes in the squid giant axon. Neuroscience 107: 527-534.

Deamer, D., Dworkin, J.P., Sandford, S.A., Bernstein, M.P., and Allamandola, L.J. 2002. The first cell membrane. Astrobiology 2: 371-381.

Fay, M.J., Walter, N.G., and Burke, J.M. 2001. Imaging of single hairpin ribozymes in solution by atomic force microscopy. RNA 7: 887-895.

Greenspan, P. and Fowler, S.D. 1985. Spectrofluorometic studies of the lipid probe, nile red. J. Lipid Res. 26: 781-789.

Hansma, H.G. and Laney, D.E. 1996. DNA binding to mica correlates with cationic radius: Assay by atomic force microscopy. Biophys. J. 70: 1933-1939.

Janas, T., Nowotarski, K., Gruszecki, W.I., and Janas, T. 2000. The effect of hexadecaprenol on molecular organization and transport properties of model membranes. Acta Biochim. Polon. 47: 661-673.

Khvorova, A., Kwak, Y.-G., Tamkun, M., Majerfeld, I., and Yarus, M. 1999. RNAs that bind and change the permeability of phospholipid membranes. Proc. Natl. Acad. Sci. 96: 10649-10654.

MacIntosh, G.C., Bariola, P.A., Newbigin, E., and Green, P.J. 2001. Characterization of Rnyl, the Saccharomyces cerevisiae member of the $\mathrm{T}_{2}$ Rnase family of RNases: Unexpected functions for ancient enzymes. Proc. Natl. Acad. Sci. 98: 1018-1023.

Marcus, Y. 1985. Ion solvation. John Wiley \& Sons, Chichester, UK.

Meuse, C.M., Krueger, S., Majkrzak, C.F., Dura, J.A., Fu, J., Connor, J.T., and Plant, A.L. 1998. Hybrid bilayer membranes in air and water: Infrared spectroscopy and neutron reflectivity studies. Biophys. J. 74: 1388-1398.

Pace, N.R. 1991. Origin of life-Facing up to the physical setting. Cell 65: 531-533.

Paula, S., Volkov, A.G., Van Hoek, A.N., Haines, T.H., and Deamer, D.W. 1996. Permeation of protons, potassium ions, and small polar molecules through phospholipid bilayers as a function of membrane thickness. Biophys. J. 70: 339-348.

Rivetti, C., Guthold, M., and Bustamante, C. 1999. Wrapping of DNA around the E. coli RNA polymerase open promoter complex. EMBO J. 18: 4464-4475.

Sedin, D.L. and Rowlen, K.L. 2001. Influence of tip size on AFM roughness measurements. Appl. Surf. Sci. 182: 40-48.

Środoń, J. 1999. Nature of mixed-layer clays and mechanism of their formation and alteration. Annu. Rev. Earth Sci. 27: 19-53.

Szostak, J.W., Bartel, D.P., and Luisi, P.L. 2001. Synthesizing life. Nature 409: 387-390.

Tomizawa, J. 1984. Control of ColE plasmid replication: The process of binding of RNA I to the primer transcript. Cell 38: 861-870.

Vlassov, A., Khvorova, A., and Yarus, M. 2001. Binding and disruption of phosholipid bilayers by supramolecular RNA complexes. Proc. Natl. Acad. Sci. 98: 7706-7711.

Walter, P., Keenan, R., and Schmitz, U. 2000. SPR-Where the RNA and membrane worlds meet. Science 287: 1212-1213.

Yang, L. and Glaser, M. 1996. Formation of membrane domains during activation of protein kinase C. Biochemistry 35: 13966-13974.

Yarus, M. 2002. Primordial genetics: Phenotype of the ribocyte. Annu. Rev. Genet. 36: 125-151. 

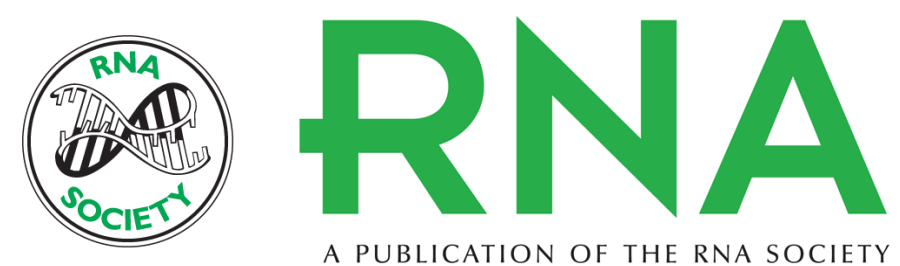

A PUBLICATION OF THE RNA SOCIETY

\section{Visualization of membrane RNAs}

\section{TADEUSZ JANAS and MICHAEL YARUS}

RNA 2003 9: 1353-1361

\section{References This article cites 20 articles, 8 of which can be accessed free at:} http://rnajournal.cshlp.org/content/9/11/1353.full.html\#ref-list-1

\section{License}
Email Alerting Receive free email alerts when new articles cite this article - sign up in the box at the Service top right corner of the article or click here.

To subscribe to $R N A$ go to:

\section{http://rnajournal.cshlp.org/subscriptions}

